\title{
Lead exposure at firing ranges-a review
}

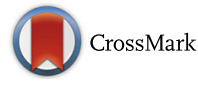

\author{
Mark A. S. Laidlaw ${ }^{1 *}$, Gabriel Filippelli ${ }^{2}$, Howard Mielke ${ }^{3}$, Brian Gulson ${ }^{4}$ and Andrew S. Ball ${ }^{1}$
}

\begin{abstract}
Background: Lead (Pb) is a toxic substance with well-known, multiple, long-term, adverse health outcomes. Shooting guns at firing ranges is an occupational necessity for security personnel, police officers, members of the military, and increasingly a recreational activity by the public. In the United States alone, an estimated 16,000-18,000 firing ranges exist. Discharge of $\mathrm{Pb}$ dust and gases is a consequence of shooting guns.

Methods: The objectives of this study are to review the literature on blood lead levels (BLLs) and potential adverse health effects associated with the shooting population. The search terms "blood lead", "lead poisoning", "lead exposure", "marksmen", "firearms", "shooting", "guns", "rifles" and "firing ranges" were used in the search engines Google Scholar, PubMed and Science Direct to identify studies that described BLLs in association with firearm use and health effects associated with shooting activities.

Results: Thirty-six articles were reviewed that included BLLs from shooters at firing ranges. In 31 studies BLLs $>10 \mu \mathrm{g} /$ $\mathrm{dL}$ were reported in some shooters, 18 studies reported BLLs $>20 \mu \mathrm{g} / \mathrm{dL}, 17$ studies $>30 \mu \mathrm{g} / \mathrm{d}$, and 15 studies BLLs > $40 \mu \mathrm{g} / \mathrm{dL}$. The literature indicates that BLLs in shooters are associated with Pb aerosol discharge from guns and air Pb at firing ranges, number of bullets discharged, and the caliber of weapon fired.

Conclusions: Shooting at firing ranges results in the discharge of $\mathrm{Pb}$ dust, elevated $\mathrm{BLLS}$, and exposures that are associated with a variety of adverse health outcomes. Women and children are among recreational shooters at special risk and they do not receive the same health protections as occupational users of firing ranges. Nearly all BLL measurements compiled in the reviewed studies exceed the current reference level of $5 \mu \mathrm{g} / \mathrm{dL}$ recommended by the U.S. Centers for Disease Control and Prevention/National Institute of Occupational Safety and Health (CDC/NIOSH). Thus firing ranges, regardless of type and user classification, currently constitute a significant and unmanaged public health problem. Prevention includes clothing changed after shooting, behavioural modifications such as banning of smoking and eating at firing ranges, improved ventilation systems and oversight of indoor ranges, and development of aiffow systems at outdoor ranges. Eliminating lead dust risk at firing ranges requires primary prevention and using lead-free primers and lead-free bullets.
\end{abstract}

Keywords: Blood, Lead, Poisoning, Shooting, Range, Firearms, Health, Effects, Expert shooter, Guns

\section{Background}

Most attention in the area of human health and guns has been rightly placed on shooting injuries and deaths [1]. However, decades of evidence indicate that substantial health risks are incurred by the shooters themselves in the form of lead exposure and subsequent poisoning. Indeed, as pointed out as early as 1994 by Ozonoff, based on high blood lead levels (BLLs) of shooters, “...

\footnotetext{
* Correspondence: mark.laidlaw@rmit.edu.au

${ }^{1}$ Centre for Environmental Sustainability and Remediation (EnSuRe), School of Science, RMIT University, PO Box 71, Bundoora, VIC 3083, Australia

Full list of author information is available at the end of the article
}

firing ranges comprise one of the largest unregulated sources of occupational or para-occupational lead exposure for adults. The perils of firearms exist at both ends of the barrel." [2]. The past two decades have brought substantial improvements in firing range environmental oversight as well as analytical capabilities to detect lead in humans, but literature evidence indicates that we fall far short of human health safety criteria in firing ranges of all types, and among occupational and recreational shooters. This review fills a gap in the literature by compiling data from a broad range of recent studies of firing range users, employees, and their families, including indoor and outdoor ranges, in an attempt to document and clarify risks by firing range use, setting, and shooting 
behaviour. The emphasis of this review is on BLLs of shooters as a marker for adverse health effects among members of the shooting population.

\section{Shooting statistics}

In the United States alone an estimated 16,000-18,000 indoor firing ranges exist which employ tens of thousands of employees [3]. An estimated 1 million law enforcement officers train at indoor firing ranges [3]. In 2011 there were approximately 270 million civilianowned firearms owners in the US and in 2007 there were approximately 650 million civilian-owned weapons globally [4] and 200 million firearms owned by nation states worldwide [5]. In the United States approximately 20 million citizens practice target shooting as a leisure activity [6]. The National Sport Shooting Foundation (NSSF) [7] stated that in 2011 in the United States there were 13,049,050 handgun shooters, 13,170,417 rifle shooters, 9,713,033 shotgun shooters, and 3,730,567 muzzleloader shooters who participated in 156,790,412 handgun shooting days, 146,652,398 rifle shooting days, $113,866,661$ shotgun shooting days and 29,042,237 muzzleloader shooting days. The global statistics regarding the number of firing ranges and shooting prevalence are not available, but it is likely there are a very large number of shooters at firing ranges. The United States Geological Survey (USGS) [8] calculated that in 2012 about 60,100 metric tonnes of lead were used in ammunition and bullets in the United States. Given that the dominant metal in bullets is lead, there are a large number of people globally who are exposed to lead from shooting at firing ranges.

\section{Source of exposure from shooting lead bullets}

There are several sources of potential lead exposure from shooting guns and firing ranges. Most bullet projectiles are made from lead, but a large amount of lead is also present in the primer, composed of approximately 35\% lead styphnate and lead peroxide (and also contains barium and antimony compounds), that ignites in a firearm barrel to provide the propulsion for the projectile [9-13]. A portion of the lead bullet disintegrates into fine fragments while passing through the gun due to misalignments of the gun barrel [9]. The lead particles, along with dust and fumes originating from the lead primer and the bullet fragments are ejected at high pressures $(18,000-$ 20,000 psi; $124-128 \mathrm{mpa}$ ) from the gun barrel, a large proportion of which occurs at right angles to the direction of fire in close proximity to the shooter [9]. The shooter can inhale fine $\mathrm{Pb}$ particulates (mainly from the primer) which constitutes the proximal exposure pathway. Fine and coarse particulates from both the primer and bullet fragments also attach to the shooters hands, clothing, and other surfaces, and can be inadvertently ingested, providing another lead exposure pathway [14, 15]. When changing targets at outdoor firing ranges shooters can be exposed to lead that has accumulated in soil dust. Additionally, the shooters can then bring these particulates back to their home and expose their families as with other lead occupational hazards.

Firing range personnel are employed by the shooting galleries, and thus also receive proximal lead exposure. They are also charged with cleaning the ranges and removing lead particulates on floors, targets, and the ventilation systems (for indoor ranges). Furthermore, they work at firing ranges for extended hours during the working week, compounding potential lead exposure.

Finally, although not the focus of this review, there are environmental impacts arising from firing ranges. Emissions in firing ranges result in the accumulation of elevated lead concentrations in surface soils [16-18]. This is concerning because lead particles do not naturally biodegrade in soil as do some contaminants such as hydrocarbons. The half-life of lead in surface soil has been estimated as approximately 700 years [19]. Therefore, if not remediated following closure, lead contaminated surface soils at firing ranges could result in lead exposures for hundreds of years. Dust from lead contaminated soil can be resuspended into the atmosphere and transported from a firing range whether outdoor or indoor [20,21]. Lead in soils and dusts at firing ranges are highly bioavailable [22]. Lead in soil could weather/ oxidize and migrate down-gradient to underlying groundwater beyond the firing range boundaries [23]. The low solubility of lead in natural water (i.e., not mining related), however, limits off-site aquatic transport. The factors most likely to affect the amount of lead carried by the groundwater in solution are $\mathrm{pH}$, depth to groundwater, soil chemistry, soil type and annual precipitation [24]. Soil-derived sediment discharged during rain events from lead contaminated firing range soils has the potential to migrate to surrounding properties and into waterways through runoff or storm drains. Wildlife [25-27], biota [28] and humans can be exposed to lead contaminated soils, sediments and airborne particulates near firing ranges. Bellinger et al. (2013) [29] provided a consensus statement about the health risks arising from lead-based ammunition in the environment.

Health outcomes associated with blood lead levels (BLLs) In 2012, The United States National Toxicology Program (NTP) published evidence regarding health effects associated with BLL exposure in adults and children [30]. For adult men and women there is "sufficient evidence" that BLLs $<10 \mu \mathrm{g} / \mathrm{dL}$ are associated with essential tremor, hypertension, cardiovascular-related mortality and electrocardiography abnormalities, and decreased kidney glomerular filtration rate. For women there is 
"sufficient evidence" that BLLs $<5 \mu \mathrm{g} / \mathrm{dL}$ are associated with reduced foetal growth. For adult men and women there is "limited evidence" that BLLs $<10 \mu \mathrm{g} / \mathrm{dL}$ were associated with psychiatric effects, decreased hearing and cognitive function, incidence of amyotrophic lateral sclerosis, and increased spontaneous abortion in women. For adults there is "limited evidence" that BLLs $<5 \mu \mathrm{g} /$ $\mathrm{dL}$ were associated with incidence of essential tremor. For children with BLLs $<5 \mu \mathrm{g} / \mathrm{dL}$ there is "sufficient evidence" of decreased academic achievement, intelligence quotient (IQ), specific cognitive measures, increased attention related behaviours, delayed puberty and reduced postnatal growth. For children with blood lead levels < $10 \mu \mathrm{g} / \mathrm{dL}$ there is "sufficient evidence" of decreased hearing. For children with BLLs $<5 \mu \mathrm{g} / \mathrm{dL}$ there is "limited evidence" of an association of decreased kidney glomerular filtration rate, and delayed puberty. For prenatal exposure with BLLs $<5 \mu \mathrm{g} / \mathrm{dL}$ there is "limited evidence" of decreases in measures of cognitive function. For prenatal lead exposure $<10 \mu \mathrm{g} / \mathrm{dL}$ there is "limited evidence" of decreased IQ, increased incidence of attention-related and problem behaviors and decreased hearing. For adult men and women there is "limited evidence" that BLLs between 15 and $20 \mu \mathrm{g} / \mathrm{dL}$ are associated with adverse sperm parameters and increased time to pregnancy in women. There is "limited evidence" that BLLs $\geq 10 \mu \mathrm{g} / \mathrm{dL}$ are associated with decreased fertility. There is "limited evidence" that spontaneous abortion occurs in female partners of men with BLLs $\geq 31 \mu \mathrm{g} / \mathrm{dL}$. However, modern exposures are orders of magnitude larger than early hominids [31] with pre-industrial blood lead levels in humans estimated at $0.016 \mu \mathrm{g} / \mathrm{dL}$ [32]. Bellinger (2011) [33] noted that adverse health effects are continually being associated with decreasing exposures.

\section{Methodology}

The search engines Google Scholar, Pubmed and Science Direct were accessed for studies that provided information about BLLs associated with firearms using the search terms "blood lead", "lead poisoning", "lead exposure", "marksmen", "firearms", "shooting", "guns", "rifle" and "firing ranges". The literature regarding the health effects specifically associated with shooting lead bullets at firing ranges was also reviewed. Studies that reported BLLs associated with shooters at firing ranges were compiled into Table 1.

\section{Results - blood lead levels at firing ranges}

The search identified 36 articles originating from 15 countries around the world published between 1975 and 2016 that included BLLs of shooters. Over half of the reports were from the U.S. The articles describe BLLs of law enforcement personnel, high school shooting coaches, and family members ranging from as young as 1 -year-old to adult men and women.

\section{Summary of blood lead levels reported}

Data from collected studies reveals the widespread occurrence of BLL by occupational and recreational shooters. The vast majority of these articles reported at least one BLL that exceeded $10 \mu \mathrm{g} / \mathrm{dL}$. About half of the studies further reported BLLs exceeding $20 \mu \mathrm{g} / \mathrm{dL}$ (18 articles), exceeding $30 \mu \mathrm{g} / \mathrm{dL}$ (17), and even exceeding > $40 \mu \mathrm{g} / \mathrm{dL}$ (15). Indeed, all 36 of the articles indicated BLLs of shooters exceeded $2 \mu \mathrm{g} / \mathrm{dL}$. Considering that the geometric mean BLL of the U.S. adult population in 2009-2010 was $1.2 \mu \mathrm{g} / \mathrm{dL}$ [34], the BLLs among shooters provide stark evidence of significant exposure, particularly to recreational shooters who do not typically selfscreen for BLL. Several key characteristics about BLLs and exposure variables arising from shooting are gleaned from the literature.

\section{Baseline and post-shooting blood lead level relationships}

Several studies focused on before-after comparisons of shooters, particularly shooters in military and police occupations, and found marked increases in BLL resulting from firing range activities. Tripathi et al. (1989) [9] measured BLLs in police cadets before, and 1,2 and 5 days after starting shooting practice, and 69 days after the start of shooting. At 69 days after the start of shooting, the BLLs of the cadets remained above baseline levels prior to shooting. Rocha et al. (2014) [35] conducted a study of BLLs of police cadets before a shooting course and 3 days after the cessation of the shooting course. The mean BLL of cadets increased from $3.3 \mu \mathrm{g} /$ $\mathrm{dL}(95 \% \mathrm{CI}=3.0-3.6 \mu \mathrm{g} / \mathrm{dL})$ before the course to $18.4 \mu \mathrm{g} / \mathrm{dL}(95 \%$ CI $16-21 \mu \mathrm{g} / \mathrm{dL}) 3$ days after completion of the course. In all cases the BLL increased significantly after the course $(p<0.001)$. Within 3 days, the BLLs of the course instructors increased from $3.6 \mu \mathrm{g} / \mathrm{dL}$ to $22.1 \mu \mathrm{g} / \mathrm{dL}$ in one case and from $7.7 \mu \mathrm{g} / \mathrm{dL}$ to $18.3 \mu \mathrm{g} / \mathrm{dL}$ in another. Fischbein et al. (1979) [36] conducted a study of 23 firearms instructors and reported that the BLLs increased measurably after firearms training. Vivante et al. (2008) [37] reported a statistically significant $(p<0.001)$ increase in BLLs of 29 Israeli soldiers from a baseline of $10.3 \pm 2.0 \mu \mathrm{g} / \mathrm{dL}$ to $18.9 \pm 3.6 \mu \mathrm{g} / \mathrm{dL}$ six weeks after training.

\section{Decline in blood lead levels after shooting events}

Several studies provide insight into the decline in BLLs following shooting events. Goldberg et al. (1991) [38] observed that average BLLs in 7 firing range instructors decreased from $45 \mu \mathrm{g} / \mathrm{dL}$ to $31 \mu \mathrm{g} / \mathrm{dL} 6$ months after a training event. George et al. (1993) [39] observed that average BLLs in 52 small bore rifle recreational shooters 
Table 1 Blood Lead Levels Observed Following Shooting Firearms at Shooting ranges

\begin{tabular}{|c|c|c|c|c|c|c|c|c|}
\hline \multirow[b]{2}{*}{ Activity } & \multirow[b]{2}{*}{ Firing } & \multirow[b]{3}{*}{ Category } & \multicolumn{3}{|c|}{ Blood Lead ( $\mu \mathrm{g} / \mathrm{dL})$} & \multirow[b]{3}{*}{ Location } & \multirow[b]{3}{*}{$\mathrm{n}$} & \multirow[b]{3}{*}{ Reference } \\
\hline & & & $\begin{array}{l}\text { Mean } \\
\text { (Median*) }\end{array}$ & SD & Range & & & \\
\hline Type & Range & & & & & & & \\
\hline \multicolumn{9}{|c|}{ Occupational } \\
\hline \multicolumn{9}{|c|}{ Indoor } \\
\hline & & Arsenal Firing Range Workers & 37.2 & 11.7 & $22-59.6$ & Taiwan & 10 & Chau et al. (1995) [80] \\
\hline & & Arsenal Other Workers & 13.3 & 5.6 & & & 98 & \\
\hline & & Firing Range and Gun Store Employees & & & $\begin{array}{l}19.9- \\
40.7\end{array}$ & California, USA & 6 & CDC (2014) [81] \\
\hline & & $\begin{array}{l}\text { Firearms instructor \& range cleaning and } \\
\text { maintenance }\end{array}$ & 88 & & & New York, USA & 1 & $\begin{array}{l}\text { Fisher-Fischbein et al. } \\
\text { (1987) [82] }\end{array}$ \\
\hline & & $\begin{array}{l}\text { Firearm Instructors Before Indoor Training: } \\
4.3 \%\end{array}$ & & & $<20$ & New York, USA & 1 & $\begin{array}{l}\text { Fischbein et al. (1979) } \\
\text { [36] }\end{array}$ \\
\hline & & $\begin{array}{l}\text { Firearm Instructors Before Indoor Training: } \\
82.6 \%\end{array}$ & & & 20-39 & & 19 & \\
\hline & & $\begin{array}{l}\text { Firearm Instructors Before Indoor Training: } \\
8.7 \%\end{array}$ & & & $40-59$ & & 2 & \\
\hline & & $\begin{array}{l}\text { Firearm Instructors Before Indoor Training: } \\
4.3 \%\end{array}$ & & & $>60$ & & 1 & \\
\hline & & Firearm Instructors After Indoor Training: 4.3\% & & & $<20$ & & 1 & \\
\hline & & $\begin{array}{l}\text { Firearm Instructors After Indoor Training: } \\
52.2 \%\end{array}$ & & & $20-39$ & & 12 & \\
\hline & & $\begin{array}{l}\text { Firearm Instructors After Indoor Training: } \\
34.8 \%\end{array}$ & & & $40-59$ & & 8 & \\
\hline & & Firearm Instructors After Indoor Training: 8.7\% & & & $>60$ & & 2 & \\
\hline & & Firearms Instructors and Officers: 42\% & & & $<40$ & New York, USA & 19 & Fischbein (1992) [83] \\
\hline & & Firearms Instructors and Officers: $34 \%$ & & & $40-60$ & & 15 & \\
\hline & & Firearms Instructors and Officers: $24 \%$ & & & $>60$ & & 11 & \\
\hline & & Firearm Instructors & 125 & 15 & & USA & 3 & $\begin{array}{l}\text { Landrigan et al. (1975) } \\
\text { [84] }\end{array}$ \\
\hline & & Male Police Officers & 5 & & $1-18.2$ & Sweden & 75 & $\begin{array}{l}\text { Löfstedt et al. (1999) } \\
\text { [85] }\end{array}$ \\
\hline & & Female Police Officers & 3.7 & & & & 3 & \\
\hline & & Full Time Employee & 77 & & & Colorado, USA & 1 & $\begin{array}{l}\text { Novotny et al. (1987) } \\
\text { [86] }\end{array}$ \\
\hline & & Full Time Employee & 59 & & & & 1 & \\
\hline & & Full Time Employee & 49 & & & & 1 & \\
\hline & & Part Time Employee & 41 & & & & 1 & \\
\hline & & Shooting range supervisor & 5.8 & 1.6 & $2.1-17.3$ & $\begin{array}{l}\text { Republic of } \\
\text { Korea }\end{array}$ & 35 & Park et al. (2016) [87] \\
\hline & & Professional shooter & 11.6 & 1.9 & $3.0-34.3$ & & 24 & \\
\hline & & Shooting range manager & 9.5 & 2.2 & $2.0-64.0$ & & 61 & \\
\hline & & All job types & 8.6 & 2.1 & $2.0-64.0$ & & 120 & \\
\hline & & Firing Range Instructors & 5.5 & 0.6 & $4.2-6.7$ & $\begin{array}{l}\text { Sao Paulo, } \\
\text { Brazil }\end{array}$ & 20 & Rocha et al. (2014) [35] \\
\hline & & Cadets - Before Training & 3.3 & 0.15 & $3.0-3.6$ & & 21 & \\
\hline & & Cadets - After Training & 18.2 & 1.5 & $16-21$ & & 21 & \\
\hline & & Instructor 1 Before Training & 3.6 & & & & 1 & \\
\hline
\end{tabular}


Table 1 Blood Lead Levels Observed Following Shooting Firearms at Shooting ranges (Continued)

\begin{tabular}{|c|c|c|c|c|c|c|}
\hline Instructor 1 After Training & 22.1 & & & & 1 & \\
\hline Instructor 2 Before Training & 7.7 & & & & 1 & \\
\hline Instructor 2 After Training & 18.3 & & & & 1 & \\
\hline Instructors & 19 & 7 & & $\begin{array}{l}\text { Amman, } \\
\text { Jordan }\end{array}$ & 31 & $\begin{array}{l}\text { Abudhaise et al. (1996) } \\
\text { [88] }\end{array}$ \\
\hline Trainees & 22.9 & 4.6 & & & 54 & \\
\hline Controls & 2.1 & 1.4 & & & unknown & \\
\hline Police Small Arms Instructors & 43.5 & 6.2 & $\begin{array}{l}33.1- \\
49.7\end{array}$ & England & 7 & Smith (1976) [89] \\
\hline US Coastguard Firearm Instructors - Pre BLL & 2.4 & 1.6 & $0.9-5.6$ & $\begin{array}{l}\text { Washington, } \\
\text { USA }\end{array}$ & 9 & Torres (2014) [90] \\
\hline US Coastguard Firearm Instructors - Post BLL & 2.3 & 1.2 & $0.9-4.6$ & & 9 & \\
\hline $\begin{array}{l}\text { US Coastguard Firearm Instructors - Pre-Post } \\
\text { BLL }\end{array}$ & 2.4 & 1.4 & $0.9-5.1$ & & 9 & \\
\hline Police Trainees: Before Training - (1/29/87) & $6.0\left(6.4^{*}\right)$ & & & Colorado, USA & 17 & Valway et al. (1989) [45] \\
\hline Police Trainees: Training - (3/3/87) & $51.1\left(51.6^{*}\right)$ & & & & 17 & \\
\hline Police Trainees: Training - (3/31/87) & $44.3\left(43.1^{*}\right)$ & & & & 17 & \\
\hline Police Trainees: Training - (5/3/87) & $39.5\left(40.0^{*}\right)$ & & & & 17 & \\
\hline Israeli Defence Soldiers Baseline & 10.3 & 2.3 & & Israel & 29 & Vivante et al. (2008) [37] \\
\hline Israeli Defence Soldiers Postexposure & 18.8 & 3.6 & & & 29 & \\
\hline Indoor Range Instructors & 8.5 & 7.6 & & Italy & unknown & $\begin{array}{l}\text { Di Lorenzo et al. (2006) } \\
\text { [91] }\end{array}$ \\
\hline \multicolumn{7}{|l|}{ Indoor/outdoor } \\
\hline FBI Instructors 1989 & 14.6 & & $9-21$ & Virginia, USA & 7 & CDC (1996) [65] \\
\hline FBI Instructors 1990 & 13.7 & & $6-27$ & & 7 & \\
\hline FBI Instructors 1991 & 7.6 & & $<4-12$ & & 14 & \\
\hline FBI Range Techs 1989 & 16.2 & & $10-24$ & & 5 & \\
\hline FBI Range Techs 1990 & 10.4 & & $6-14$ & & 5 & \\
\hline FBI Range Techs 1991 & 13.6 & & $8-28$ & & 5 & \\
\hline US Special Operations Forces - Year 2000 & 13.90 & & & USA & 255 & $\begin{array}{l}\text { Mancuso et al. (2008) } \\
\text { [92] }\end{array}$ \\
\hline US Special Operations Forces - Year 2005 & 6.80 & & & & & \\
\hline Adult Target Shooters - Occupational - 21.6\% & & & $<25$ & New York, USA & 26 & $\begin{array}{l}\text { Gelberg and DePersis } \\
\text { (2009) [93] }\end{array}$ \\
\hline Adult Target Shooters - Occupational - 50.8\% & & & $25-39$ & & 61 & \\
\hline Adult Target Shooters - Occupational - 25\% & & & $40-59$ & & 30 & \\
\hline Adult Target Shooters - Occupational - 2.5\% & & & $60+$ & & 3 & \\
\hline \multicolumn{7}{|l|}{ Outdoor } \\
\hline Range Instructors & 6.7 & 5.3 & & Italy & unknown & $\begin{array}{l}\text { Di Lorenzo et al. (2006) } \\
\text { [91] }\end{array}$ \\
\hline Firing Range Instructors Before Training & 41 & 10 & $28-66$ & California, USA & 7 & $\begin{array}{l}\text { Goldberg et al. (1991) } \\
\text { [38] }\end{array}$ \\
\hline Firing Range Instructors After Training & 45 & 14 & $28-70$ & & 7 & \\
\hline $\begin{array}{l}\text { Firing Range Instructors } 6 \text { Months After } \\
\text { Training }\end{array}$ & 31 & 5 & $28-38$ & & 7 & \\
\hline Soldiers Before Basic Training & $<0.29$ & & & Israel & 22 & $\begin{array}{l}\text { Greenberg et al. (2016) } \\
\text { [94] }\end{array}$ \\
\hline Soldiers After Basic Training & 1.17 & 1.73 & & & 22 & \\
\hline
\end{tabular}


Table 1 Blood Lead Levels Observed Following Shooting Firearms at Shooting ranges (Continued)

\begin{tabular}{|c|c|c|c|c|c|c|}
\hline Soldiers After Advanced Training & 3.92 & 1.99 & & & 22 & \\
\hline Body guards & 8.10 & 2.70 & $3.0-14.4$ & Mexico & 26 & Madrid et al. (2016) [41] \\
\hline Instructors & 7.60 & 1.40 & $6.6-8.6$ & & 2 & \\
\hline Shooting Range Caretakers & 40.65 & 15.63 & $\begin{array}{l}29.6- \\
51.7\end{array}$ & & 2 & \\
\hline Administrative & 4.20 & 1.20 & $2.7-6.5$ & & 9 & \\
\hline Security & 4.90 & 1.20 & $3.0-7.3$ & & 9 & \\
\hline Services & 6.50 & 3.60 & $3.1-14.2$ & & 10 & \\
\hline Elite Group & 6.40 & 2.60 & $4.2-11.4$ & & 6 & \\
\hline All Categories & 7.60 & 6.80 & $2.7-51.7$ & & 64 & \\
\hline Howitzer Operators - Baseline & 5.5 & & $\begin{array}{l}4.33- \\
6.73\end{array}$ & $\begin{array}{l}\text { Oklahoma, } \\
\text { USA }\end{array}$ & 20 & Smart et al. (1994) [40] \\
\hline Howitzer Operators - After 3rd Exercise & 20.1 & & $\begin{array}{l}17.94- \\
22.44\end{array}$ & & 20 & \\
\hline Howitzer Operators - 8 Weeks After Exposure & 11.9 & & $\begin{array}{l}9.92- \\
14.02\end{array}$ & & 12 & \\
\hline Shooting Instructor \#1 Non-Jacketed bullets & 24.02 & & & Virginia, USA & 1 & Tripathi et al. (1991) [46] \\
\hline $\begin{array}{l}\text { Shooting Instructor \#1 Copper Jacketed } \\
\text { Bullets }\end{array}$ & 21.95 & & & & 1 & \\
\hline Shooting Instructor \#2 Non-Jacketed bullets & 14.08 & & & & 1 & \\
\hline $\begin{array}{l}\text { Shooting Instructor \#2 Copper Jacketed } \\
\text { Bullets }\end{array}$ & 13.04 & & & & 1 & \\
\hline $\begin{array}{l}\text { Outdoor Firing Range Police Cadets: Baseline - } \\
\text { Day } 0\end{array}$ & 6 & 1.1 & $5-8$ & Virginia, USA & 7 & Tripathi et al. (1989) [9] \\
\hline Outdoor Firing Range Police Cadets: Day 1 & 6 & 1.0 & $5-8$ & & 7 & \\
\hline Outdoor Firing Range Police Cadets: Day 2 & 11 & 2.1 & $7-14$ & & 7 & \\
\hline Outdoor Firing Range Police Cadets: Day 5 & 15 & 5.4 & $9-26$ & & 7 & \\
\hline Outdoor Firing Range Police Cadets: Day 69 & 9 & 2.8 & $6-14$ & & 7 & \\
\hline \multicolumn{7}{|l|}{ Non-Occupational } \\
\hline \multicolumn{7}{|l|}{ Indoor } \\
\hline High School Shooting Coach & 44 & & & Alaska, USA & 1 & CDC (2005) [56] \\
\hline $\begin{array}{l}\text { Firing Range A (School Range) - Students } \\
\text { Aged 15-17 years }\end{array}$ & 24.3 & & $\begin{array}{l}21.0- \\
31.0\end{array}$ & & 7 & \\
\hline $\begin{array}{l}\text { Firing Range B (Commercial) - Students Aged } \\
13-16 \text { years }\end{array}$ & 2.1 & & & & 8 & \\
\hline $\begin{array}{l}\text { Firing Range C (Volunteer Run) - Students } \\
\text { Aged 15-19 years }\end{array}$ & 18.5 & & $5-37$ & & 24 & \\
\hline $\begin{array}{l}\text { Firing Range D (School Range) - Students } \\
\text { Aged } 14-17 \text { years }\end{array}$ & 8.9 & & $3.0-14.0$ & & 7 & \\
\hline $\begin{array}{l}\text { Firing Range } E \text { (Volunteer Run) - Students } \\
\text { Aged } 7-17 \text { years }\end{array}$ & 7.6 & & $2-13$ & & 20 & \\
\hline Shooter & $9.2^{*}$ & & $2.7-52.1$ & Germany & 129 & $\begin{array}{l}\text { Demmeler et al. (2009) } \\
\text { [44] }\end{array}$ \\
\hline Shooter: Q1 200 rounds/month & $8.7^{*}$ & & $2.8-31.4$ & & 27 & \\
\hline Shooter: Q2 200-399 rounds/month & $9^{*}$ & & $2.7-31.5$ & & 28 & \\
\hline Shooter: Q3 400-680 rounds/month & $11.8^{*}$ & & $2.9-37.5$ & & 29 & \\
\hline Shooter: Q4 > 680 rounds/month & $13.8^{*}$ & & $3.7-52.1$ & & 23 & \\
\hline Airguns & $3.3^{*}$ & & $1.8-12.7$ & & 20 & \\
\hline
\end{tabular}


Table 1 Blood Lead Levels Observed Following Shooting Firearms at Shooting ranges (Continued)

\begin{tabular}{|c|c|c|c|c|c|c|}
\hline Airguns and .22 caliber & $8.7^{*}$ & & $1.4-17.2$ & & 15 & \\
\hline .22 Ir bore \& large caliber & $10.7^{*}$ & & $2.7-37.5$ & & 51 & \\
\hline Large caliber handgun & $10^{*}$ & & $2.8-32.6$ & & 32 & \\
\hline $\begin{array}{l}\text { IPSC (International Practical Shooting } \\
\text { Federation) }\end{array}$ & $19.2^{*}$ & & $3.2-52.1$ & & 11 & \\
\hline \multicolumn{2}{|l|}{$\begin{array}{l}\text { Adult Target Shooters - Both Occupational \& Non- } \\
\text { Occupational - } 1.5 \%\end{array}$} & & $<25$ & New York, USA & 2 & \multirow[t]{4}{*}{$\begin{array}{l}\text { Gelberg and DePersis } \\
\text { (2009) [93] }\end{array}$} \\
\hline \multicolumn{2}{|l|}{$\begin{array}{l}\text { Adult Target Shooters - Both Occupational \& Non- } \\
\text { Occupational - } 71.3 \%\end{array}$} & & $25-39$ & & 92 & \\
\hline \multicolumn{2}{|l|}{$\begin{array}{l}\text { Adult Target Shooters - Both Occupational \& Non- } \\
\text { Occupational - } 20.9 \%\end{array}$} & & $40-59$ & & 27 & \\
\hline \multicolumn{2}{|l|}{$\begin{array}{l}\text { Adult Target Shooters - Both Occupational \& Non- } \\
\text { Occupational - } 6.2 \%\end{array}$} & & $60+$ & & 8 & \\
\hline $\begin{array}{l}\text { Small Bore Rifle Shooter - End of Indoor } \\
\text { Season }\end{array}$ & 54.65 & & & New Zealand & 52 & George et al. (1993) [39] \\
\hline $\begin{array}{l}\text { Small Bore Rifle Shooter - Preseason } \\
\text { Following Year }\end{array}$ & 33.13 & & & & 37 & \\
\hline Shooting Range \#2 & 10.5 & 7 & & South Africa & 30 & Mathee et al. (2017) [15] \\
\hline Shooting Range \#3 & 16.1 & 9.8 & & & 17 & \\
\hline Shooting Range \#4 & 19.2 & 16.3 & & & 14 & \\
\hline Child (Age 1 year) - "index case" & 46 & & & USA & 1 & Moore (1995) [95] \\
\hline Mother - (Age 25 years) & 11 & & & & 1 & \\
\hline Father - (Age 25 years) & 15 & & & & 1 & \\
\hline Sibling - (Age 4) & 14 & & & & 1 & \\
\hline Sibling - (Age 2) & 19 & & & & 1 & \\
\hline Cousin - (Age 6) & 28 & & & & 1 & \\
\hline Cousin - (Age 8) & 31 & & & & 1 & \\
\hline Aunt - (Age 37) & 30 & & & & 1 & \\
\hline $\begin{array}{l}\text { Child (Age } 1 \text { year.) "index case": } 3 \text { Months } \\
\text { After Intervention }\end{array}$ & 19 & & & & 1 & \\
\hline $\begin{array}{l}\text { Mother - (Age } 25 \text { years): } 3 \text { Months After } \\
\text { Intervention }\end{array}$ & 8 & & & & 1 & \\
\hline $\begin{array}{l}\text { Father - (Age } 25 \text { years): } 3 \text { Months After } \\
\text { Intervention }\end{array}$ & $\begin{array}{l}\text { Not } \\
\text { Measured }\end{array}$ & & & & 1 & \\
\hline Sibling - (Age 4): 3 Months After Intervention & 11 & & & & 1 & \\
\hline Sibling - (Age 2): 3 Months After Intervention & 12 & & & & 1 & \\
\hline Cousin- (Age 6): 3 Months After Intervention & 13 & & & & 1 & \\
\hline Cousin - (Age 8): 3 Months After Intervention & 13 & & & & 1 & \\
\hline Aunt - (Age 37): 3 Months After Intervention & 13 & & & & 1 & \\
\hline Indoor Shooting Range Employee Spouse & 6 & & & Colorado, USA & 1 & $\begin{array}{l}\text { Novotny et al. (1987) } \\
\text { [86] }\end{array}$ \\
\hline Indoor Shooting Range Employee Spouse & 11 & & & & 1 & \\
\hline Indoor Shooting Range Employee Spouse & 6 & & & & 1 & \\
\hline Recreational Shooters & $29^{*}$ & & $24-45$ & Germany & 7 & $\begin{array}{l}\text { Ochsmann et al. (2009) } \\
\text { [6] }\end{array}$ \\
\hline $\begin{array}{l}\text { Competitive Marksmen - Adolescents Aged } \\
14-16 \text { years }\end{array}$ & 21.3 & & $18-28$ & $\begin{array}{l}\text { Massachusetts, } \\
\text { USA }\end{array}$ & 4 & Shannon (1999) [57] \\
\hline Shooters before indoor shooting season & $10.6^{*}$ & & $3.2-17.6$ & Sweden & 22 & $\begin{array}{l}\text { Svensson et al. (1992) } \\
\text { [96] }\end{array}$ \\
\hline
\end{tabular}


Table 1 Blood Lead Levels Observed Following Shooting Firearms at Shooting ranges (Continued)

\begin{tabular}{|c|c|c|c|c|c|c|}
\hline Shooters after indoor shooting season & $13.8^{*}$ & & $6.9-28.8$ & & 22 & \\
\hline $\begin{array}{l}\text { Airgun Shooters before indoor shooting } \\
\text { season }\end{array}$ & $9.1^{*}$ & & $4.7-17.9$ & & 21 & \\
\hline Airgun Shooters after indoor shooting season & $8.4^{*}$ & & $2.0-22.2$ & & 21 & \\
\hline Archers before shooting season & $6.1^{*}$ & & $2.7-9.2$ & & 13 & \\
\hline Archers after shooting season & $5.6^{*}$ & & $3.1-8.7$ & & 13 & \\
\hline $\begin{array}{l}\text { Shooter: shot } 600 \text { rounds } 3 \text { times per week } \\
\text { for past } 4 \text { months }\end{array}$ & 43.5 & & & $\begin{array}{l}\text { United } \\
\text { Kingdom }\end{array}$ & 1 & White (1996) [97] \\
\hline \multicolumn{7}{|l|}{ Indoor/Outdoor } \\
\hline Shooter & 45 & 6.3 & $35-47$ & $\begin{array}{l}\text { Connecticut, } \\
\text { USA }\end{array}$ & 6 & Cook et al. (2015) [98] \\
\hline $\begin{array}{l}\text { Irregular Frequency Indoor and Outdoor } \\
\text { Shooter }\end{array}$ & 6.7 & & & Australia & 1 & Gulson et al. (2002) [99] \\
\hline Archers who also shot guns & 7.8 & 10.26 & & South Africa & 15 & Mathee et al. (2017) [15] \\
\hline $\begin{array}{l}\text { Gun shooters only (excluding archers and } \\
\text { employees) }\end{array}$ & 12.13 & 10.18 & & & 62 & \\
\hline $\begin{array}{l}\text { Gun shooters who also work at the shooting } \\
\text { range }\end{array}$ & 16.3 & 7.83 & & & 8 & \\
\hline $\begin{array}{l}\text { All gun shooters (including archers and } \\
\text { employees) }\end{array}$ & 11.9 & 10.15 & & & 85 & \\
\hline Less than monthly & 8.4 & 5.5 & & & 23 & \\
\hline$>$ monthly, but less than weekly & 11.8 & 8.7 & & & 35 & \\
\hline$>$ weekly, but $<3$ times per week & 14.3 & 15.2 & & & 21 & \\
\hline$>3$ times per week & 12.5 & 9.7 & & & 7 & \\
\hline Pistol Sport Shooters & 10.76 & 8.28 & & $\begin{array}{l}\text { Helsinki, } \\
\text { Finland }\end{array}$ & 20 & $\begin{array}{l}\text { Asa-Mäkitaipale et al. } \\
\text { (2009) [42] }\end{array}$ \\
\hline \multicolumn{7}{|l|}{ Outdoor } \\
\hline Archery Range & 4.5 & 2.7 & & South Africa & 14 & Mathee et al. (2017) [15] \\
\hline Shooting Range \#1 & 7 & 4.3 & & & 12 & \\
\hline Practised archery only & 3.3 & 2.25 & & & 31 & \\
\hline
\end{tabular}

declined from $54.7 \mu \mathrm{g} / \mathrm{dL}$ at the end of the indoor season to $33.1 \mu \mathrm{g} / \mathrm{dL}$ in 37 of the shooters by the preseason of the following year. Smart et al. (1994) [40] observed that average BLLs of 20 howitzer operators declined from $20.1 \mu \mathrm{g} / \mathrm{dL}$ to $11.9 \mu \mathrm{g} / \mathrm{dL}$ in 12 operators 8 weeks after ending the firing exercise. Tripathi et al. (1989) [9] observed that BLLs of 7 outdoor firing range police cadets had a baseline average of $6 \mu \mathrm{g} / \mathrm{dL}$ prior to commencing shooting training and an average BLL of $15 \mu \mathrm{g} / \mathrm{dL}$ at the end of training 5 days later. At follow up 69 days after training, the average BLL was $9 \mu \mathrm{g} / \mathrm{dL}$. Thus, the results indicate that BLLs following shooting events can remain elevated for a considerable time after cessation of shooting, especially for participants with higher BLLs.

\section{Association between blood lead levels and bullets fired, caliber of weapon, copper jacketed or unjacketed bullets and air lead levels}

Several characteristics such as shooting frequency, caliber of the gun, type of bullet, and air lead at firing ranges have been studied. Each of these variables relate to BLLs and can also be associated with environmental issues at firing ranges.

\section{BLLs and frequency of shooting activity}

Most studies reviewed indicate a strong positive correlation between the use frequency of shooters at firing ranges and their BLLs. Madrid et al. (2016) [41] reported that BLLs were higher $(p<0.001)$ in individuals who participated in greater than 12 shooting practice sessions per year $(8.3 \pm$ $2.4 \mu \mathrm{g} / \mathrm{dL}$ ) compared with controls who shot less than 12 times per year $(5.2 \pm 2.5 \mu \mathrm{g} / \mathrm{dL})$. Tripathi et al. (1989) [9] observed a positive association between the total number of rounds fired and BLLs $(\mathrm{r}=0.84 ; p<0.02)$ and personalbreathing-zone air lead levels $(r=0.92 ; p<0.001)$. Air lead levels were also correlated with BLLs $(r=0.85 ; p<0.02)$. Asa-Mäkitaipale et al. (2009) [42] reported a correlation between BLLs and bullets fired during the last month $(r=0.71$; $\mathrm{p} 0.001)$ and the past year $(r=0.55 ; \mathrm{p} 0.012)$. Betancourt (2012) [43] observed a linear relationship between air lead 
exposure and total number of rounds fired by caliber of weapon used.

\section{Blood lead and gun caliber}

Relationships between BLL and caliber of firearms have also been described. Demmeler et al. (2009) [44] observed that the larger the caliber of the weapon, the higher the shooters BLL. The following median BLLs were reported: airguns - $3.3 \mu \mathrm{g} / \mathrm{dL}$ (range 1.8-12.7 $\mu \mathrm{g}$ / $\mathrm{dL}$ ); 0.22 caliber weapons - $8.7 \mu \mathrm{g} / \mathrm{dL}$ (range 1.4$17.2 \mu \mathrm{g} / \mathrm{dL}) ; 0.22$ caliber and large caliber handguns (9 mm or larger) - $10.7 \mu \mathrm{g} / \mathrm{dL}$ (range $2.7-37.5 \mu \mathrm{g} / \mathrm{dL}$ ); and large caliber handguns - $10.0 \mu \mathrm{g} / \mathrm{dL}$ (range 2.8$32.6 \mu \mathrm{g} / \mathrm{dL}$ ). Demmeler et al. [44] also reported that shooters belonging to the International Practical Shooting Confederation (IPSC) had the highest median BLL of $19.2 \mu \mathrm{g} / \mathrm{dL}$. Additionally, studies indicated a positive correlation between cumulative air lead exposure in firing ranges and BLL of shooters [40, 45].

\section{BLLs and copper jacketed vs. unjacketed bullets}

Tripathi et al. (1991) [46] compared the BLLs in firearm instructors using copper jacketed and non-jacketed bullets. One shooting instructor exhibited BLLs of $24.0 \mu \mathrm{g} /$ $\mathrm{dL}$ and $22.0 \mu \mathrm{g} / \mathrm{dL}$ using non-jacketed bullets and copper-jacketed bullets, respectively. A second instructor exhibited BLLs of $14.1 \mu \mathrm{g} / \mathrm{dL}$ and $13.0 \mu \mathrm{g} / \mathrm{dL}$ using non-jacketed bullets and copper-jacketed bullets, respectively.

\section{BLLs and air lead}

Elevated BLLs especially arising from indoor firing ranges are the result of the greater absorption of lead from inhalation compared with ingestion and dermal absorption. For example, the amount of absorption of ingested lead by adults under non-fasting conditions ranges from 3 to $10 \%$ and in young children from 40 to $50 \%$ whereas inhaled lead lodging deep in the respiratory tract seems to be absorbed equally and totally, regardless of chemical form [47]. As shooting involves generation of extremely fine particles and gases, the high rate of absorption logically results in elevated BLLs. Outdoor ranges, presumably well-ventilated by natural flow and large air volumes, do not necessarily prevent lead exposure from shooting activities. The following sections discuss the implications of the results.

\section{Discussion}

The results in Table 1 must be evaluated in the context of BLL recommendations, special need populations, air lead measured at firing ranges, and prevention. The use of ventilation to manage exposure at firing ranges and prevent lead exposure of shooters is appraised.
Blood lead level recommendations from public and occupational health communities

Several United States (US) governmental agencies have developed recommendations regarding BLLs. The Centers for Disease Control and Prevention (CDC) makes health recommendations to protect public health whereas the National Institute for Occupational Safety and Health (NIOSH) and the Occupational Safety and Health Administration (OSHA) focus on worker health. The trend for BLL recommendations has been declining over several decades since regulations were first established.

\section{CDC and NIOSH}

The CDC [34] makes the following statement regarding recommended BLLs in adults:

"In 2015, NIOSH designated $5 \mu \mathrm{g} / \mathrm{dL}$ (five micrograms per deciliter) of whole blood, in a venous blood sample, as the reference blood lead level for adults. An elevated BLL is defined as a $B L L \geq 5 \mu \mathrm{g} / d L$. This case definition is used by the ABLES program, the Council of State and Territorial Epidemiologists (CSTE), and CDC's National Notifiable Diseases Surveillance System (NNDSS). Previously (i.e. from 2009 until November 2015), the case definition for an elevated $B L L$ was a $B L L \geq 10 \mu \mathrm{g} / d L$. The U.S. Department of Health and Human Services recommends that BLLs among all adults be reduced to $<10 \mu \mathrm{g} / \mathrm{dL}$. The U.S. Occupational Safety and Health Administration (OSHA) Lead Standards require workers to be removed from lead exposure when BLLs are equal or greater than $50 \mu \mathrm{g} / \mathrm{dL}$ (construction industry) or $60 \mu \mathrm{g} / \mathrm{dL}$ (general industry) and allow workers to return to work when the BLL is below $40 \mu \mathrm{g} / d L$.... OSHA Lead Standards give the examining physician broad flexibility to tailor special protective procedures to the needs of individual employees. Therefore, the most current guidelines for management of leadexposed adults should be implemented by the medical community at the current CDC/NIOSH reference BLL of $5 \mathrm{\mu g} / \mathrm{dL}$. Recommendations for medical management are available from the Association of Occupational and Environmental Clinics, California Department of Public Health, and the Council of State and Territorial Epidemiologist (CSTE) Occupational Health Surveillance Subcommittee."

\section{Council of state and territorial epidemiologists (CSTE)}

The CSTE is an organization of member states and territories representing public health epidemiologists in the United States. The CSTE [48] makes the following recommendations actions for various blood lead levels in adults (Table 2): 
Table 2 Council of State and Territorial Epidemiologists Management Recommendations for Adult Blood Lead Levels

\begin{tabular}{ll}
\hline $\begin{array}{l}\text { Blood Lead Level } \\
(\mu \mathrm{g} / \mathrm{dL})\end{array}$ & Management Recommendations \\
\hline$<5$ & No action needed \\
& Monitor BLL if ongoing exposure \\
& Discuss health risks \\
& Minimize exposure \\
& Consider removal for pregnancy and certain \\
& medical conditions \\
& Monitor BLL \\
& Decrease exposure \\
& Remove from exposure for pregnancy \\
& Consider removal for certain medical conditions or \\
& BLL > $>$ \\
& 10 for an extended period of time \\
& Monitor BLL \\
& Remove from exposure for pregnancy \\
& Remove from exposure if repeat BLL in 4 weeks \\
& remains $>$ 20 \\
& Annual lead medical exam recommended \\
& Remove from exposure \\
& Prompt medical evaluation \\
& Remove from exposure \\
& Prompt medical evaluation \\
& Consider chelation with significant symptoms \\
& Remove from exposure \\
& Urgent medical evaluation \\
& Chelation may be indicated \\
&
\end{tabular}

Ideally, recommendation triggering immediate cessation of exposure at shooting ranges should not be based on a single blood lead level measurement. The duration of an elevated BLL over multiple BLL measurements should determine the nature of the intervention. Current public health recommendations call first for education and attention to risk factors that can mitigate future exposures.

\section{Occupational health and safety administration (OSHA) and the new science-based recommendations}

For occupational shooters and firing range workers, the U.S. OSHA Lead Standards require general industry workers to be removed from lead exposure when BLLs are equal or greater than $60 \mu \mathrm{g} / \mathrm{dL}$, and allows them to return to work when their BLL is below $40 \mu \mathrm{g} / \mathrm{dL}$ [34]. Based on the recommended BLLs by the $\mathrm{CDC} / \mathrm{NIOSH}$ [34], the CSTE [48] and the comprehensive compilation of health effects of low level lead exposure by the NTP [30], the OSHA regulation that allows workers to return to work with BLLs greater than $40 \mu \mathrm{g} / \mathrm{dL}$ seems nonsensical as a health risk avoidance guideline, and should be lowered in line with the Council of State and Territorial Epidemiologists (CSTE) recommendations, as shown in Council of state and territorial epidemiologists (CSTE).

\section{Special needs of women and children}

Lead exposure of women and children have special characteristics that must be taken into account. The needs relate to the effect of lead on future generations. For women the needs are related to the effect of lead on the developing fetus and post-natal exposure associated with breast-feeding. For children the special needs for low exposure are related to the extraordinary sensitivity of the developing organs of children. These concerns indicate the need for a margin of safety.

\section{The special lead risks of women}

The risk to women exposed to lead at firing ranges is of particular concern because, once absorbed, a proportion of the lead is deposited in the skeleton and more than $90 \%$ of lead in adults is stored in their bones. Bone storage takes place because due to their similar ionic radius and charge lead is substituted for calcium. Furthermore, when a woman becomes pregnant the fetus requires calcium and, depending on the dietary intakes, a proportion of calcium is derived from remodelling of the bones. Skeletal lead stores are released from the remodelling exposing the fetus during critical development windows [49-51]. Even modestly elevated BLL's have been associated with serious neurological disorders such as autism [52]. Lead released from a woman's bones during pregnancy is associated with foetal developmental problems [53]. Another consideration for female shooters is that when their BLL becomes elevated, they can pass the lead on to their children through breast milk [54, 55]. Given the known lead contamination at firing ranges, intending-to- conceive, pregnant women, and nursing mothers should curtail exposure from shooting activities (employed in the security, military and police, and recreational shooters) and observe precautionary prevention.

\section{Health risks related to children shooters}

The CDC (2005) [56] reported that children (aged 7-18) shooting bullets at multiple firing ranges in Alaska exhibited highly elevated BLLs (see Table 1). Shannon (1999) [57] reported that children (aged 14-16) who were competitive marksmen exhibited an average BLL of $21.3 \mu \mathrm{g} / \mathrm{dL}$ (range 18-28 $\mu \mathrm{g} / \mathrm{dL}$ ). Blood lead levels observed in children from shooting activities are within the range known to cause long-term detrimental health effects [30]. Exposure of young females to lead is of particular concern because it is stored in their bones and 
can then be transferred to their developing fetus many years later when they become pregnant [49-51].

\section{Health-related lead issues and law enforcement personnel} Law enforcement includes a number of services to protect and ensure the safety of citizens and the community. The public requires law enforcement personnel to be "calm, cool and collected" when in service conducting their duties. However, the adverse health effects, especially on the nervous system that are associated with elevated BLLs arising from firearm use are inconsistent with these ideals.

\section{Air lead levels at firing ranges}

The OSHA 8-h air lead time weighted average (TWA) action level is $30 \mu \mathrm{g} / \mathrm{m}^{3}$ and the OSHA permissible exposure limit (PEL) is $50 \mu \mathrm{g} / \mathrm{m}^{3}$ [58]. The California Department of Public Health Occupational Lead Poisoning Prevention Program (CDPH-OLPPP) recommended $8 \mathrm{~h}$ TWA PEL is 0.5 to $2.1 \mu \mathrm{g} / \mathrm{m}^{3}$ [59]. Based on this guideline, the CDPH-OLPPP states "At a PEL of $0.5 \mu \mathrm{g} / \mathrm{m}^{3}$, 95\% of workers would have a BLL less than $5 \mu \mathrm{g} / d L$ over a 40 year working lifetime. At a PEL of $2.1 \mu \mathrm{g} / \mathrm{m}^{3}, 95 \%$ of workers would have a BLL less than $10 \mu \mathrm{g} / \mathrm{dL}$ and $57 \%$ would have a BLL less than $5 \mu \mathrm{g} / d L$ over their working lifetime." Wang et al. (2016) [60] conducted a review of studies of airborne lead concentrations and possible exposure at firing ranges (Additional file 1). Wang et al. [60] found that the OSHA $8 \mathrm{~h}$ TWA PEL is exceeded in many studies, and even more noteworthy, the California PEL is exceeded in all of the studies. It must be noted that the recommended PEL and action levels are not the only paths to controlling lead exposures.

\section{Biomonitoring and primary prevention}

Kosnett et al. (2007) [61] recommend that: "individuals be removed from occupational lead exposure if a single blood lead concentration exceeds 30 microg/dL or if two successive blood lead concentrations measured over a 4week interval are $>$ or $=20 \mathrm{microg} / \mathrm{dL}$. Removal of individuals from lead exposure should be considered to avoid long-term risk to health if exposure control measures over an extended period do not decrease blood lead concentrations to $<10$ microg/dL or if selected medical conditions exist that would increase the risk of continued exposure." A more conservative approach are the recommendations by CSTE in Council of state and territorial epidemiologists (CSTE). A critical issue is that biomonitoring is not primary prevention. Biomonitoring only assesses the degree of exposure and potential health damage after exposure has taken place. Primary prevention requires curtailing lead exposure and maintenance of air quality. Several steps have been proposed above to minimize lead exposure. Recommendations to prevent occupational lead poisoning by shooters are provided by U.S. Government [62]. The recommendations appear as topics in school rifle team programs [63].

One of the challenges in a biomonitoring program is the frequency which shooters should have their BLLs monitored. The Australian organisation Safe Work Australia has recently carefully made recommendations for multiple scenarios of blood lead testing frequency for workers exposed to lead in the work place [64]. Similar BLL testing frequency recommendations could be adopted for shooters exposed to lead in occupational settings such as law enforcement, military, security and shooting range workers. Recreational shooters that shoot frequently could voluntarily use these blood lead testing frequency recommendations as a guide if they wanted to protect their health.

\section{Potential health risks from 'take home lead'}

In contrast to occupational environments where work clothes should not be taken home, lead dust can adhere to shooters clothes and potentially contaminate vehicles and homes. The CDC (1996) [65] measured carpet dust lead concentrations in FBI student dormitory rooms and in 14 non-student dormitory rooms at a firing range and training facility. They observed that student dormitory rooms had significantly higher lead levels than non-student dormitory rooms, suggesting that the FBI students were contaminating their living quarters with lead. 'Take home lead' has been described mostly for occupational settings [66-68] but given the fine particle nature and lead concentrations of dust associated with shooting, the 'take home lead' pathway of exposure from shooting must be recognized and curtailed.

\section{Prevention of lead aerosols with ventilation improvements}

The air lead table from Wang et al. 2016 [60] (Additional file 1) and the National Research Council [69] are the only compilations of air $\mathrm{Pb}$ levels at shooting ranges that were identified. Wang et al. do not discuss the ventilation practices in the various studies that may account for lower air lead levels. A 1975 NIOSH study found that at all 9 ranges studied, the air lead guideline was exceeded at the time $\left(200 \mu \mathrm{g} / \mathrm{m}^{3}\right)$ [70]. A $2009 \mathrm{NIOSH}$ review describes a case study on air lead exposure of law enforcement trainees and reports that the mean airborne lead concentration of $>2000 \mu \mathrm{g} / \mathrm{m}^{3}$ was reduced by 94 $97 \%$ to $60-120 \mu \mathrm{g} / \mathrm{m}^{3}$ but this was still above the OSHA PEL of $50 \mu \mathrm{g} / \mathrm{m}^{3}$. Commercial ventilation companies claim they can meet guidelines (i.e. Camfil air filters) but no published studies supporting this achievement at firing ranges were located.

There is a "lack of evidence" gap in the literature demonstrating that ventilation systems can maintain air lead levels at indoor ranges below the current OSHA $(50 \mu \mathrm{g} /$ 
$\left.\mathrm{m}^{3}\right)$ or California $\left(0.5-2.2 \mu \mathrm{g} / \mathrm{m}^{3}\right)$ guideline. The literature gap raises questions about whether or not the guidelines can actually be achieved, especially the California guideline. Further, as discussed in Special needs of women and children, meeting the guideline does not necessarily provide a margin of safety from lead exposure.

\section{Primary prevention requires eliminating lead in primers and bullets}

Lead from projectile primers is a significant proximal source of lead exposure and uptake. The development of primers is described by Brede et al. [71]. During the $19^{\text {th }}$ century primers were composed of mercury fulminate; however, the mercury fulminate was found to be too toxic to shooters. In the early $20^{\text {th }}$ Century, Dynamit Nobel developed the primer SINOXID which was formulated with lead and became a universal primer. By “... the 1960s exposure of shooters and firing range supervisors to lead reached intolerably high levels, as evidenced by the elevated blood lead values [71]." Dynamit Nobel developed SINTOX, a Pb-free (as well as $\mathrm{Sb}$ and $\mathrm{Ba}$ free) primer [71]. However, the results of some tests of the lead-free primers have proven disappointing, with significant variations in ignition timing, peak blast pressure, higher barrel frictions, and reliability in different climate conditions, compared with their lead-based equivalents [72]. The performance of lead-free primers are being tested by the U.S. Department of Defense (DoD) and North Atlantic Treaty Organisation (NATO) to reduce exposure of personnel to known lead sources [73].

Despite the critical observations, there is lead-free ammunition on the market. SINTOX is NATO approved and outlets for lead-free ammunition are available [74, 75]. Some states are taking the issue seriously and require lead-free (or non-toxic "NT") ammunition at firing ranges [76]. Widespread acceptance of the need to replace lead must take place, and until this happens one of the most significant health risks to shooters will remain lead-rich primers.

"Green bullets" have also been proposed as a preventative measure that could minimize lead exposure to participants and the environment. These bullets consist of copper rather than lead bullets. Bismuth has been proposed as a substitute for lead bullets but its environmental health impacts are poorly understood [77]. It is clear that firing lead-free bullets results in dramatic decreases in airborne lead exposures at firing ranges [78]. The use of copper-jacketed lead bullets does not appear to be a solution to a reduction in lead exposure because it results in only minor reductions in BLLs (see Tripathi et al. (1991, Table 1) [46]. The United States Department of Defence (DoD) is aware of the health threat posed by lead exposure from small arms [69] and efforts are underway to test and replace lead in both primer and bullets [73, 79].

Table 1 provides evidence-based information about the BLL sensitivity of shooters to lead dust at firing ranges. The major gap in preventing risk of lead exposure at firing ranges are the fundamental lead-bearing materials used for the explosive power and bullet projectiles. Primary prevention requires eliminating all lead materials in primers and bullets in order to end the dispersal of lead dust at firing ranges.

\section{Conclusions}

Shooting lead bullets at firing ranges results in elevated BLLs at concentrations that are associated with a variety of adverse health outcomes and the topic of health risk is an ongoing topic of study. Of major concern is the number of women and children among recreational shooters, who are not afforded similar health protections as occupational users of firing ranges. Nearly all BLL measurements compiled in the reviewed studies exceed the level of $5 \mu \mathrm{g} / \mathrm{dL}$ recommended by the U.S. CDC/ $\mathrm{NIOSH}$, and thus firing ranges, regardless of type and user classification, constitute a significant and currently largely unmanaged public health concern. Primary prevention of this risk requires development of lead-free primers and projectile bullets. Prevention includes better oversight of ventilation systems in indoor ranges and development of airflow systems at outdoor ranges, protective clothing that is changed after shooting, and cessation of smoking and eating at firing ranges. The mismatch between what is recommended for individuals by the U.S. CDC is in stark contrast to the allowable levels for occupational exposure, and there are no real systematic biomonitoring programs for firing range users to measure cumulative health effects caused by persistent low and even high-level lead exposure. Recreational shooters and the general public are provided no legal protections from lead exposures at firing ranges. In conclusion, while the past two decades have brought substantial improvements in analytical capabilities to detect lead in humans the literature evidence indicates that we fall far short of human health safety criteria in firing ranges of all types, and among occupational and recreational shooters.

\section{Additional file}

Additional file 1: Summary of Studies on Airborne Lead Exposure and Concentration from Shooting Activities, by Chronological Orders (modified from Wang et al., 2016). (DOCX $19 \mathrm{~kb}$ )

\section{Abbreviations}

ABLES: Adult blood lead and epidemiology surveillance; BLLs: Blood lead levels; CDC: Centers for disease control and prevention; $\mathrm{CDPH}-$

OLPPP: California department of public health occupational lead poisoning 
prevention program; CSTE: Council of state and territorial epidemiologists: DOD: Department of defence; IPSC: International practical shooting confederation; IQ: Intelligence quotient; NATO: North atlantic treaty organisation; $\mathrm{NIOSH}$ : National institute for occupational safety and health; NNDSS: National notifiable diseases surveillance system; NSSF: National sports shooting foundation; NT: Non-toxic; NTP: National toxicology program; OSHA: Occupational safety and health administration; Pb: Lead; PEL: Permissible exposure level; US: United States; USGS: United States geological survey

\section{Acknowledgements}

We are very thankful to the three reviewers whose valuable comments substantially improved this manuscript. Mark Laidlaw thanks RMIT University for providing his funding through the Vice Chancellors Postdoctoral Fellowship Scheme. We would also like to thank Elizabeth O'Brien of the Lead Group Inc. in Sydney who stimulated the idea for this review. Thanks to Christopher Gonzales for assistance with Primary prevention requires eliminating lead in primers and bullets

\section{Funding}

Mark A.S. Laidlaw received funding from the RMIT University Vice Chancellor's Postdoctoral Fellowship. Funding for the remaining authors was from their salaries at their respective universities.

\section{Availability of data and material}

All data generated or analysed during this study are included in this published article.

\section{Authors' contributions}

$\mathrm{ML}$ conducted the initial literature review and wrote the first draft. HM, GF, $B G$, and $A B$ reviewed and edited the subsequent drafts of the manuscript. All authors read and approved the final manuscript.

\section{Competing interests}

The authors declare they have no actual or potential competing financial interests.

\section{Consent for publication}

Not Applicable.

\section{Ethics approval and consent to participate}

Not Applicable.

\section{Publisher's Note}

Springer Nature remains neutral with regard to jurisdictional claims in published maps and institutional affiliations.

\section{Author details}

${ }^{1}$ Centre for Environmental Sustainability and Remediation (EnSuRe), School of Science, RMIT University, PO Box 71, Bundoora, VIC 3083, Australia.

${ }^{2}$ Department of Earth Sciences and Center for Urban Health, Indiana University-Purdue University Indianapolis (IUPUI), Indianapolis, IN, USA. ${ }^{3}$ Tulane University School of Medicine, New Orleans, LA, USA. ${ }^{4}$ Department of Environmental Sciences, Macquarie University, Sydney, Australia.

\section{Received: 26 October 2016 Accepted: 30 March 2017}

\section{Published online: 04 April 2017}

\section{References}

1. Swedler DI, Simmons MM, Dominici F, Hemenway D. Firearm prevalence and homicides of law enforcement officers in the United States. Am J Public Health. 2015;105:2042-8.

2. Ozonoff D. Lead on the range. Lancet. 1994:343:6-7.

3. Beaucham C, Page E, Alarcon WA, Calver GM, Methner M, Schoonover TM Indoor firing ranges and elevated BLLs - United States, 2002- 2013. MMWR Morb Mortal Wkly. 2014;63:347-51. https://www.cdc.gov/mmwr/preview/ mmwrhtml/mm6316a3.htm Accessed 17 Jan 2017

4. Small Arms Survey (SAS). Estimating civilian owned firearms. In: Small arms survey research notes, number 9, September 2011. 2011. http://www. smallarmssurvey.org/fileadmin/docs/H-Research_Notes/SAS-Research-Note9.pdf. Accessed 2 Sept 2016.
5. Small Arms Survey (SAS). 2007. Completing the Count: Civilian Firearms. Graduate Institute Geneva. 2007. http://www.smallarmssurvey.org/ publications/by-type/yearbook/small-arms-survey-2007.html. Accessed 2 Sept 2016.

6. Ochsmann E, Göen T, Schaller KH, Drexler H. Lead-still a health threat for marksmen. Int J Hyg Environ Health. 2009;212:557-61.

7. National Shooting Sports Foundation (NSSF). Target shooting in America. 2013. http://www.nssf.org/PDF/research/TargetShootingInAmericaReport.pdf Accessed 2 Sept 2016

8. U.S. Geological Survey (USGS): Mineral industry surveys: lead in January 2013. http://minerals.usgs.gov/minerals/pubs/commodity/lead/mis-201301lead.pdf Accessed 23 Oct 2016.

9. Tripathi RK, Llewellyn GC. Deterioration of air quality in firing ranges: A review of airborne lead exposures. In: Biodeterioration Research 1989;445-457.

10. Haw CK, Jayaprakasha PT, Hooib YC, Abdullaha AF. Health concern on lead encountered during firing practices: a review. Health Environ J. 2010;1:24-9. http://www.hej.kk.usm.my/pdf/HEJVol.1No.2/Article04.pdf Accessed 29 Oct 2016.

11. Basu S. Formation of gunshot residues. J Forensic Sci. 1982;27:72-91.

12. Caddy B, Meng HH. Gunshot residue analysis - a review. J Forensic Sci. 1997:42:553-70.

13. Romolo FS, Margot P. Identification of gunshot residue: a critical review. Forensic Sci Int. 2001;119:195-211.

14. Dalby O, Butler D, Birkett JW. Analysis of Gunshot Residue and Associated Materials-A Review. J Forensic Sci. 2010;55:924-43.

15. Mathee $A$, de Jager $P$, Naidoo $S$, Naicker $N$. Exposure to lead in south African shooting ranges. Environ Res. 2017;153:93-8.

16. Murray K, Bazzi A, Carter C, Ehlert A, Kopec M, Richardson J, Sokol H. Distribution and mobility of lead in soils at an outdoor shooting range. Soil Sediment Contam. 1997:6:79-93.

17. Chen M, Daroub SH, Ma LQ, Harris WG, Cao X. Characterization of lead in soils of a rifle/pistol shooting range in central Florida, USA. Soil Sediment Contam. 2002;11:1-7.

18. Fayiga AO, Saha UK. Soil pollution at outdoor shooting ranges: health effects, bioavailability and best management practices. Environ Pollut. 2016:216:135-45.

19. Semlali RM, Dessogne JB, Monna F, Bolte J, Azimi S, Navarro N, Denaix L, Loubet M, Chateau C, Van Oort F. Modeling lead input and output in soils using lead isotopic geochemistry. Environ Sci Technol. 2004;38:1513-21.

20. Laidlaw MA, Zahran S, Mielke HW, Taylor MP, Filippelli GM. Re-suspension of lead contaminated urban soil as a dominant source of atmospheric lead in Birmingham, Chicago, Detroit and Pittsburgh, USA. Atmos Environ. 2012;49:302-10.

21. Laidlaw MA, Zahran S, Pingitore N, Clague J, Devlin G, Taylor MP. dentification of lead sources in residential environments: Sydney Australia. Environ Pollut. 2014:184:238-46.

22. Bannon DI, Drexler JW, Fent GM, Casteel SW, Hunter PJ, Brattin WJ, Major MA. Evaluation of small arms range soils for metal contamination and lead bioavailability. Environ Sci Technol. 2009;43(24):9071-6.

23. Hardison DW, Ma LQ, Luongo T, Harris WG. Lead contamination in shooting range soils from abrasion of lead bullets and subsequent weathering. Sci Total Environ. 2004:328:175-83.

24. United States Environmental Protection Agency (USEPA). Best Management Practices for Lead at Outdoor Shooting Ranges. EPA-902-B-01-001. Revised 2005. https://www.epa.gov/sites/production/files/documents/epa_bmp.pdf. Accessed 17 Jan 2017.

25. Lewis LA, Poppenga RJ, Davidson WR, Fischer JR, Morgan KA. Lead toxicosis and trace element levels in wild birds and mammals at a firearms training facility. Arch Environ Contam Toxicol. 2001;41:208-14.

26. Johnson MS, Major MA, Casteel SW. Lead accumulation in woodchucks (Marmotamonax) at small arms and skeet ranges. Ecotoxicol Environ Saf. 2004:59:232-6.

27. Pain DJ, Fisher IJ, Thomas VG. A global update of lead poisoning in terrestrial birds from ammunition sources. In: Ingestion of lead from spent ammunition: implications for wildlife and humans. 2009. https://www.peregrinefund.org/ subsites/conference-lead/PDF/0108\%20Pain.pdf Accessed 1 Oct 2016.

28. Labare MP, Butkus MA, Riegner D, Schommer N, Atkinson J. Evaluation of lead movement from the abiotic to biotic at a small-arms firing range Environ Geol. 2004:46:750-4

29. Bellinger DC, Burger J, Cade TJ, Cory-Slechta DA, Finkelstein M, Hu H, et al. Health risks from lead-based ammunition in the environment. EHP. 2013; 121(6):A 178-9. doi:10.1289/ehp.1306945. 
30. National Toxicology Program. NTP monograph: health effects of Low-level lead. 2012. https://ntp.niehs.nih.gov/pubhealth/hat/noms/lead/index.html Accessed 1 September 2016.

31. Patterson C, Ericson J, Manea-Krichten M, Shirahata H. Natural skeletal levels of lead in Homo sapiens sapiens uncontaminated by technological lead. Sci Total Environ. 1991;107:205-36.

32. Flegal AR, Smith DR. Lead levels in preindustrial humans. N Engl J Med. 1992:326:1293-4.

33. Bellinger $\mathrm{D}$. The protean toxicities of lead: New chapters in a familiar story. Int J Environ Res Public Health. 2011:8:2593-628. doi:10.3390/ijerph8072593.

34. Centers for Disease Control and Prevention (CDC). Adult Blood Lead Epidemiology and Surveillance (ABLES) - Program Description. https://www. cdc.gov/niosh/topics/ables/description.html Accessed 18 Jan 2017.

35. Rocha ED, Sarkis JE, Maria de Fátima HC, dos Santos GV, Canesso C. Occupational exposure to airborne lead in Brazilian police officers. Int J Hyg Environ Health. 2014;217:702-4.

36. Fischbein A, Rice C, Sarkozi L, Kon SH, Petrocci M, Selikoff IJ. Exposure to lead in firing ranges. JAMA. 1979;241(11):1141-4

37. Vivante A, Hirshoren N, Shochat T, Merkel D. Association between acute lead exposure in indoor firing ranges and iron metabolism. Isr Med Assoc J. 2008;10:292.

38. Goldberg RL, Hicks AM, O'Leary LM, London S. Lead exposure at uncovered outdoor firing ranges. J Occup Med. 1991;33:718-9.

39. George PM, Walmsley TA, Currie D, Wells JE. Lead exposure during recreational use of small bore rifle ranges. New Zealand Med J. 1993;106:422-4.

40. Smart DA, Parmer DL, Young JY, Hoffmann F, Langford RE. 1994. Biological responses of $155 \mathrm{~mm}$ Howitzer crewmen to airborne lead. Walter Reed Army Inst Res Washington DC. C; 1994 Oct 21 https://scholar.google.com/ scholar?q=Biological+responses+of+155mm+Howitzer+crewmen+to +airborne+lead\&btnG=\&hl=en\&as_sdt=0\%2C24 Accessed 1 Oct 2016.

41. Madrid GA, Téllez-Cárdenas L, Juárez-Pérez CA, Haro-García LC, MercadoGarcía A, Gopar-Nieto R, Cabello-López A. Blood lead determinants and the prevalence of neuropsychiatric symptoms in firearm users in Mexico. Int J Occup Med Environ Health. 2016;1:219-28.

42. Asa-Mäkitaipale S, Jehkonen M, Uitti J, Vilkki J. Memory functions in recreational pistol sport shooters: does lead matter? Environ Health Insights. 2009;3:13.

43. Betancourt JR. Determinants of airborne lead exposure during special operations training for United States Marines. Masters Thesis. San Diego State University. 2012. http://sdsu-dspace.calstate.edu/bitstream/handle/ 10211.10/3454/Betancourt_Juan.pdf?sequence=1 Accesssed 18 Jan 2017

44. Demmeler M, Nowak D, Schierl R. High BLLs in recreational indoor-shooters. Int Arch Occup Environ Health. 2009;82:539-42.

45. Valway SE, Martyny JW, Miller JR, Cook M, Mangione EJ. Lead absorption in indoor firing range users. Am J Public Health. 1989;79:1029-32.

46. Tripathi RK, Sherertz PC, Llewellyn GC, Armstrong CW. Lead exposure in outdoor firearm instructors. Am J Public Health. 1991;81:753-5.

47. Agency for Toxic Substances and Disease Registry (ATDSR). Tox Guide For Lead. CAS\# 7439-92-1. 2007. https://www.atsdr.cdc.gov/toxguides/toxguide13.pdf Accessed 19 Jan 2017

48. Council of State and Territorial Epidemiologists (CSTE). Management Guidelines for Blood Lead Levels in Adults. http://c.ymcdn.com/sites/www. cste.org/resource/resmgr/OccupationalHealth/ ManagementGuidelinesforAdult.pdf Accessed 18 Jan 2017.

49. Gulson BL, Mizon KJ, Korsch MJ, Palmer JM, Donnelly JB. Mobilization of lead from human bone tissue during pregnancy and lactation: a summary of long-term research. Sci Total Environ. 2003;303:79-104

50. Gulson B, Mizon K, Korsch M, Taylor A. Revisiting mobilisation of skeletal lead during pregnancy based on monthly sampling and cord/maternal blood lead relationships confirm placental transfer of lead. Arch Toxicol 2015;1-2.

51. Gulson B, Taylor A, Eisman J. Bone remodeling during pregnancy and postpartum assessed by metal lead levels and isotopic concentrations. Bone. 2016:89:40-51.

52. Kim KN, Kwon HJ, Hong YC. Low-level lead exposure and autistic behaviors in school-age children. Neurotoxicology. 2016;53:193-200

53. Bellinger D, Leviton A, Sloman J, Rabinowitz M, Needleman $H$, Waternaux C. Low-level lead exposure and Children's cognitive function in the preschool years. Pediatrics. 1991;87:219-27.

54. Gulson BL, Jameson CW, Mahaffey KR, Mizon KJ, Patison N, Law AJ, Korsch MJ, Salter MA. Relationships of lead in breast milk to lead in blood, urine, and diet of the infant and mother. Environ Health Perspect. 1998;106(10):667.

55. Li PJ, Sheng YZ, Wang QY, Gu LY, Wang YL. Transfer of lead via placenta and breast milk in human. Biomed Environ Sci. 2000;13:85-9.
56. Centers for Disease Control (CDC). Lead exposure from indoor firing ranges among students on shooting teams - Alaska, 2002-2004. MMWR Morb Mortal Wkly Rep. 2005;54:577-9. http://www.cdc.gov/mmwr/preview/ mmwrhtml/mm5423a1.htm. Accessed 1 Sept 2016.

57. Shannon M. Lead poisoning in adolescents who are competitive marksmen. N Engl J Med. 1999:341(11):852.

58. Occupational Safety and Health Administration (OSHA). Regulation standards - 29CFR: Standard Number 1910.1025. 2016. https://www.osha. gov/pls/oshaweb/owadisp.show_document?p_table=STANDARDS\&p_id= 10030. Accessed 17 Jan 2017).

59. California Department of Public Health (CDPH-OLPPP), 2013 http://www.cdph. ca.gov/programs/olppp/documents/leadstdpelrec.pdf. Accessed 10 Jan 2017.

60. Wang J, Li H, Bezerra ML. Assessment of shooter's task-based exposure to airborne lead and acidic gas at indoor and outdoor ranges. Journal of Chemical Health and Safety. 2016(in press). http://dx.doi.org/10.1016/j.jchas. 2016.11.003

61. Kosnett MJ, Wedeen RP, Rothenberg SJ, Hipkins KL, Materna BL, Schwartz $\mathrm{BS}, \mathrm{Hu} \mathrm{H}$, Woolf A. Recommendations for medical management of adult lead exposure. Environ Health Perspect. 2007:115:463-71.

62. DHHS (NIOSH). Preventing occupational exposures to lead and noise at indoor firing ranges publication number 2009-136. 2009. https://www.cdc. gov/niosh/docs/2009-136/pdfs/2009-136.pdf Accessed 17 Jan 2017.

63. Connecticut Department of Public Health (CPDH). Fact sheet: shoot a healthy 200: lead safety for rifle teams. 2011. http://www.ct.gov/dph/lib/ dph/environmental_health/eoha/pdf/rifle_team_lead_safety_fact_sheet.pdf Accessed 17 Jan 2017.

64. Safe Work Australia. Decision regulation impact statement managing risks associated with lead in the workplace: blood lead removal levels and workplace exposure standard. 2016. http://www.safeworkaustralia.gov.au/ sites/SWA/about/Publications/Documents/990/decision-regulation-impactstatement-managing-risks-associated-with-lead-in-the-workplace.pdf. Accessed 15 March 2017.

65. Centers for Disease Control (CDC). Health hazard evaluation report 910346-2572 FBI academy Quantico, Virginia. 1996. https://www.cdc.gov/ niosh/hhe/reports/pdfs/1991-0346-2572.pdf Accessed 10 September 2016.

66. Gulson BL, Mizon KJ, Korsch MJ, Howarth D. Importance of monitoring family members in establishing sources and pathways of lead in blood. Sci Total Environ. 1996;188:173-82.

67. Chiaradia M, Gulson BL, MacDonald K. Contamination of houses by occupationally-exposed workers from a lead-zinc-copper mine and impact on blood leads in the families. Occup Environ Med. 1997;54:117-24.

68. James M, Gulson BL. Engine reconditioning workshops: lead contamination and the potential risk for workers - a pilot study. Occup Environ Med. 1999;66:429-31.

69. National Research Council (NRC). Potential health risks to DoD firing-range personnel from recurrent lead exposure. Washington: National Academies Press. ISBN 978-0-309-26736-6, 2012. 178 pp. http://www.eenews.net/assets/ 2012/12/04/document daily 01.pdf. Accessed 10 Jan 2017.

70. Anania, T.L., Seta, J.A. 1975. Lead exposure and design considerations for indoor firing ranges. OHEW Publication No. (NIOSH) 76-130. https://www. cdc.gov/niosh/docs/76-130/

71. Brede U, Hagel R, Redeker KH, Weuter W. Primer compositions in the course of time: from black powder and SINOXID to SINTOX compositions and SINCO booster. Propellants Explos Pyrotech. 1996:21:113-1 17.

72. Courtney E, Courtney A, Summer P, Courtney M. Performance testing of lead free primers: blast waves, velocity variations, and environmental testing. 2014. https://arxiv.org/abs/1410.6390 Accessed 20 Jan 2017.

73. TechLink. MIC lead-free primer for ammunition and cartridge/propellant actuated devices. 2016. http://techlinkcenter.org/summaries/mic-lead-free-primerammunition-and-cartridgepropellant-actuated-devices Accessed 20 Jan 2017.

74. Lead-free hunting. Lead free bullet types. 2016. http://www.leadfreehunting. com/. Accessed 20 Jan 2017.

75. Tampa Bay Conservation League. Environmentally-friendly (100\% lead-free) ammunition. 2014. http://conservetampabay.blogspot.com/2014/08/ environmentally-friendly-100-lead-free.html. Acccessed 20 Jan 2017.

76. New York State Brochure. Aim at lead safety. 2016. https://www.health.ny. gov/environmental/lead/target shooting/. Accessed 20 Jan 2017.

77. Jenkins, Joel A., "Viability of Bismuth as a Green Substitute for Lead in Jacketed 357 Magnum Revolver Bullets" 2015.Graduate Theses and Dissertations. http://scholarcommons.usf.edu/etd/5511. Accessed 1 Oct 2016

78. Bonanno J, Robson MG, Buckley B, Modica M. Lead exposure at a covered outdoor firing range. Bull Environ Contam Toxicol. 2002;68:315-23. 
79. Cameron EJ. Comparative Analysis of Airborne Chemical Exposure to Air Force Small Arms Range Instructors. Air Force Inst Tech Wright-Patterson AFB. OH School Engineering Management 2006. http://www.dtic.mil/dtic/tr/ fulltext/u2/a450111.pdf. Accessed 7 Sept 2016.

80. Chau TT, Chen WY, Hsiao TM, Liu HW. Chronic lead intoxication at an indoor firing range in Taiwan. J Toxicol Clin Toxicol. 1995;33:371-2.

81. Centers for Disease Control and Prevention (CDC). Health hazard evaluation report: lead exposure at a firing range and gun store. HETA 2013-0119-3219. 2014. https://www.cdc.gov/niosh/hhe/reports/pdfs/2013-0119-3219.pdf. Accessed 17 Jan 2017.

82. Fisher-Fischbein J, Fischbein A, Melnick HD, Bardin CW. Correlation between biochemical indicators of lead exposure and semen quality in a leadpoisoned firearms instructor. JAMA. 1987;257:803-5.

83. Fischbein A. Lead poisoning: I. Some clinical and toxicological observations on the effects of occupational lead exposure among firearms instructors. Isr J Med Sci. 1992;28:560-72.

84. Landrigan PJ, McKinney AS, Hopkins LC, Rhodes WW, Price WA, Cox DH. Chronic lead absorption: result of poor ventilation in an indoor pistol range. JAMA. 1975;234:394-7.

85. Lofstedt H, Selden A, Storeus L, Bodin L. Blood lead in Swedish police officers. Am J Ind Med. 1999;35:519-22.

86. Novotny $T$, Cook M, Hughes J, Lee SA. Lead exposure in a firing range. Am J Public Health. 1987;77:1225-6.

87. Park WJ, Lee SH, Lee SH, Yoon HS, Moon JD. Occupational lead exposure from indoor firing ranges in Korea. J Korean Med Sci. 2016;31:497-501.

88. Abudhaise BA, Alzoubi MA, Rabi AZ, Alwash RM. Lead exposure in indoor firing ranges: environmental impact and health risk to the range users. Int J Occup Med Environ Health. 1996;9:323-9.

89. Smith DL. Lead absorption in police small-arms instructors. Occup Med. 1976;26:139-40.

90. Torres MA. Characterizing Lead Exposure at a US Coast Guard Indoor Firing Range. 2014. (Doctoral dissertation, University of Washington). https://dlib.lib. washington.edu/researchworks/bitstream/handle/1773/26339/Torres_ washington_02500_13279.pdf?sequence=1\&isAllowed=y. Accessed 1 Oct 2016.

91. Di Lorenzo L, Borraccia V, Corfiati M, Mantineo GA, Petrillo MR, Soleo L. [Exposure to low doses of inorganic lead and arterial pressure among fire arm instructors of the Italian state police]. G Ital Med Lav Ergon. 2006;28:194-5.

92. Mancuso JD, McCoy J, Pelka B, Kahn PJ, Gaydos JC. The challenge of controlling lead and silica exposures from firing ranges in a special operations force. Mil Med. 2008;173:182-6.

93. Gelberg $\mathrm{KH}$, DePersis R. Lead exposure among target shooters. Arch Environ Occup Health. 2009;64:115-20.

94. Greenberg N, Frimer R, Meyer R, Derazne E, Chodick G. Lead exposure in military outdoor firing ranges. Mil Med. 2016;181:1121-6.

95. Moore RS, Ducatman AM. Home on the range: childhood lead exposure due to family occupation. Arch Pediatr Adolesc Med. 1995;149:1276-7.

96. Svensson BG, Schütz A, Nilsson A, Skerfving S. Lead exposure in indoor firing ranges. Int Arch Occup Environ Health. 1992:64:219-21.

97. White SA, Narula AA. A complication of indoor pistol shooting. J Laryngol Otol. 1996:110:663-4.

98. Cook JM, Sakr CJ, Redlich CA, DeLoreto AL. Elevated BLLs related to the use of firearms. Occup Environ Med. 2015;57:136-8.

99. Gulson BL, Palmer JM, Bryce A. Changes in blood lead of a recreational shooter. Sci Total Environ. 2002;293:143-50.

\section{Submit your next manuscript to BioMed Central and we will help you at every step:}

- We accept pre-submission inquiries

- Our selector tool helps you to find the most relevant journal

- We provide round the clock customer support

- Convenient online submission

- Thorough peer review

- Inclusion in PubMed and all major indexing services

- Maximum visibility for your research

Submit your manuscript at www.biomedcentral.com/submit 